\title{
PERFORMANCE ANALYSIS FOR THE USE OF ADAPTIVE BEAMFORMING IN WIRELESS PACKET NETWORKS
}

\author{
Farrokh Rashid-Farrokhi ${ }^{\dagger}$ and K.J. Ray Liu* ${ }^{*}$ \\ $\dagger$ Bell Labs, Lucent Technologies, USA \\ 791 Holmdel-Keyport Rd., Holmdel, NJ 07726, USA \\ * University of Maryland at College Park and Institute for System research \\ College Park, MD 20742, USA
}

\section{ABSTRACT}

In this work we use the interference reduction capability of smart antennas to increase the throughput and reduce the delay in wireless packet networks. We provide a theoretical framework to calculate the throughput where we use adaptive arrays in slotted ALOHA and CDMA slotted ALOHA systems. We will show that by deploying adaptive arrays at base stations, we can improve the throughput both in the request access and in the traffic channels. We also consider the effect of call admission at high traffic load. Finally, we propose an adaptive beamforming architecture for multi-code CDMA (MC-CDMA) and investigate the improvement in delay and throughput in those systems.

\section{INTRODUCTION}

Throughput in random assignment systems is low due to collisions. In ALOHA scheme, packets are transmitted as they are generated [1], and since transmissions are not coordinated, a considerable number of packets experience collision. This effect is lessened in slotted ALOHA, where packets are transmitted within a time slot. The duration of each time slot is equal to the length of a packet. If more than one packet are transmitted in the same time slot, collision occurs and all the users involved in collision will retransmit their packets after a random delay.

Throughput in CDMA systems can be increased by spreading and error correction to combat multiuser interference [2]. DS-CDMA slotted ALOHA has both advantages of using statistical multiplexing of CDMA and random access property of slotted ALOHA schemes. In [2] the performance of DS-CDMA was evaluated. In that work it was assumed that each mobile is assigned a unique PN sequence and there is a distinct receiver for each mobile at its assigned base station. The effect of forward error correction on the performance of DS-CDMA is considered in [3].

We consider the problem of beamforming in packet networks where antenna arrays are deployed at receivers. We use preambles to acquire packets and adapt the beam pattern. We consider two cases: limited number of beamformers, and preassigned beamformers. When the number of beamformers is limited, users select them by a random access scheme. If a beamformer is chosen by only one user, its packet is acquired; otherwise, it is lost and the transmitter will retransmit it after a random delay. We aiso consider the case where a unique beamformer is assigned to each user at call setup. The performance of a network with call admission is also evaluated, where the number of active transmitters are controlled to reduce the cochannel interference. We provide an analytical framework to evaluate the delay and throughput for the above cases.

Multicode CDMA (MC-CDMA) has been proposed for multi-media wireless communications [8]. The combination of MC-CDMA and Distributed Queuing Request Update Multiple Access (DQRUMA) is considered to improve traffic in CDMA networks [5]. These systems support different QoS (Quality of Service) for mobiles with different data rates. The integrated slot by slot assignment of users, in those systems, provides an efficient bandwidth on demand platform, which is well suited for our beamforming algorithms. We will show that by using smart antennas, we are able to increase both uplink and downlink capacity significantly in those systems.

This paper is organized as follows: In Section III we introduce beamforming for wireless packet systems. In Section IV we present a framework for performance evaluation of slotted ALOHA and CDMA slotted ALOHA. systems. A multi-code CDMA system using adaptive arrays is proposed in Section V. Finally, performance of the above systems is evaluated in Section VI through numerical study.

\section{SYSTEM MODEL}

Consider a network of $N$ mobiles transmitting to a base station by a slotted ALOHA multiple access scheme, where base stations use adaptive arrays with $M$ elements. At the beginning of each packet there is an acquisition preamble with enough error correction to support a large number of concurrent transmissions. There are also a beamforming preamble and a data payload as shown in Fig. 1. The beamforming preamble is used as a training sequence to calculate the weight vectors, and the beampattern is kept constant during the packet transmission. If changes in the channel response and the location of mobiles are significant during this period, packets have to be broken into smaller sizes. The received signal at each antenna array is given by

$$
\mathbf{x}(t)=\sum_{i=1}^{N} \sqrt{P_{j}} s_{j}(t) \mathbf{a}_{j}+\mathbf{n}(t),
$$

where $\mathbf{n}(t)$ is the thermal noise at the receiver array and $\mathbf{a}_{j}$ is the array response to the $j$ th transmitter. $s_{j}(t)$ and $P_{j}$ are the $j$ th transmitted signal and power, respectively. At the base station, there is a dedicated beamformer for each transmitter. As illustrated in Fig. 2, the beamformer output is the weighted sum of its inputs: $y_{i}=\mathbf{w}_{i}^{H} \mathbf{x}$. Denote the beamforming preamble by $d_{i}(t)$. The beamforming 
vector is calculated by the following minimization

$$
\mathbf{w}_{i}=\arg \min _{\mathbf{w}_{i}} E\left\{\left|d_{i}(t)-\mathbf{w}_{i}^{H} \mathbf{x}_{i}(t)\right|^{2}\right\}
$$

It can be shown that Weiner-Hopf solution $\left(\hat{\mathbf{w}}_{i}=\Phi_{i}^{-1} \mathbf{p}_{i}\right)$ maximizes the SINR (Signal to Interference and Noise Ratio) at the output of beamformer, where $\mathbf{p}_{i}=E\left\{\mathbf{x}_{i} d_{i}^{*}\right\}$, and $\phi_{i}=E\left\{\mathbf{x}_{i} \mathbf{x}_{i}^{H}\right\}$. When the number of transmitted packets is less than the number of array elements, the beamformer rejects the interference by placing nulls at the directions of these transmitters. In this case, the SINR at the beamformer output increases significantly and transmitted packets will be received with a small error probability. If the number of packets are more than the number of array elements, all the transmitted packets are lost due to low SINR.

CDMA systems require small SINR due to processing gain. As a result, they are able to receive multiple packets in the same slot even without adaptive antennas. Adaptive arrays can be employed to increase the capacity even further. In a CDMA slotted ALOHA system we may transmit a training sequence in the packet header or through the pilot channel if there is one. By using the method proposed in [7], we perform the beamforming without transmitting a training sequence. The block diagram of this system is similar to Fig. 2 where matched filter blocks are spreading code match filters. In this method we estimate the correlation matrix of the signal before matched filter, $\Phi=E\left\{\mathbf{x x}^{H}\right\}$, and the correlation matrix of the signal after the matched filter, $\Phi_{o}=E\left\{\mathbf{z z}^{H}\right\}$. The array response can be calculated by

$$
\Phi_{o}=\Phi_{i n}+P \mathbf{a}_{i} \mathbf{a}_{i}^{H}, \quad \text { and } \quad \Phi_{i n}=\frac{\eta}{\eta-1}\left(\Phi-\frac{1}{\eta} \Phi_{o}\right),
$$

where $\eta$ is the processing gain. The optimal weight vector is given by [7]: $\mathbf{w}_{i}=\frac{\Phi_{i n}^{-1} \mathbf{a}_{i}}{\mathbf{a}_{i}^{H} \Phi_{i n}^{-1} \mathbf{a}_{i}}$. The SINR can be written as $S I N R=\eta P \mathbf{a}_{i}^{H} \Phi_{i n}^{-1} \mathbf{a}_{i}$. When the number of users is large, SINR at the beamformer output is given by [7]

$$
S I N R=\frac{\eta M P}{\sigma^{2}+(N-1) P} .
$$

It is assumed that the average received power for all users is equal to $P$. Using this method, we can update the beamforming coefficients during the packet transmission to track the mobility or channel response variations.

\section{SLOTTED ALOHA PERFORMANCE EVALUATION}

We use the diagram shown in Fig. 3 to model both slotted ALOHA, and CDMA slotted ALOHA systems. In these systems the length of each packet is equal to the time slot duration and each transmitter is synchronized to its receiver such that all the packets are received within a time slot. We use antenna arrays to increase the throughput of slotted ALOHA networks. In these systems, beamforming preambles are used to adjust the beam pattern to the direction of the desired transmitter. We have to provide a beamformer at the receiver for each transmitted packet. In general we have a limited number of beamformers, and as a result a limited number of preambles in the system. Preambles are selected by mobiles randomly. If more than one mobile chooses the same preamble, contention occurs and all packets are lost. Only those packets which use distinct preambles are acquired. We also consider the case where preambles are preassigned and as a result all the transmitted packets are acquired.

The acquired packets experience multiuser interference and some of them are received successfully. An immediate feedback is sent to the successful transmitters at the end of the time slot. Those users who have not transmitted successfully will not generate new packets and will try to retransmit until their backlogged packets are successfully received. As the number of active users grows the multiuser interference increases, which decreases the throughput in high traffic rate. In order to prevent this effect we limit the number of active users by a call admission mechanism, in which only a selected set of packets receive the transmit permission.

The performance of a network is a function of the number of bocklogged users and active users. It is also a function of the retransmission probability $p_{R}$ and the new packet transmission $p_{0}$, as well as the number of available preambles, $\mathrm{K}$. We assume that transmitters choose any of the $K$ preambles independently and by random. Only packets with distinct preambles will be acquired. The conditional probability of acquiring $a$ packets, given that $n$ packets are transmitted, denoted by $P_{A}(a \mid n)$, can be calculated by

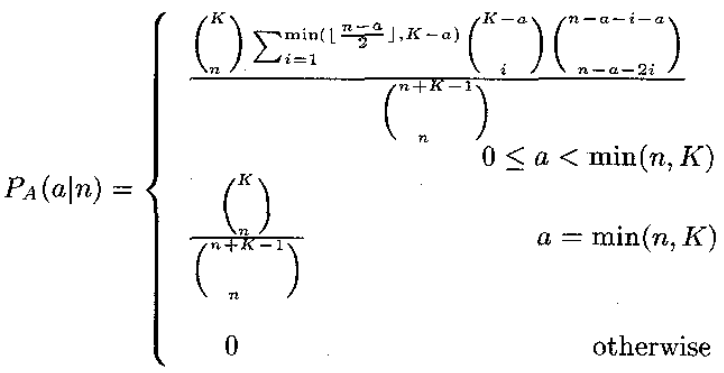

Some of the acquired packets are lost due to multiuser interference, with probability $P_{E}(n)$. Therefore, the conditional probability of successful transmission of $s \geq a$ packets given the total number of transmitted packets $n$, denoted by $P_{S}(s \mid n)$, is given by

$P_{S}(s \mid n)=\sum_{a=s}^{\min (K, n)}\left({ }_{s}^{a}\right)\left[1-P_{E}(n)\right]^{s} P_{E}(n)^{a-s} P_{A}(a \mid n)$.

In the special case that there are enough preambles, we assign a specific preamble to each user at call setup. Packet loss is then only due to multiuser interference, and the conditional probability of successful transmission is given by

$$
P_{S}(s \mid n)=\left({ }_{s}^{n}\right)\left[1-P_{E}(n)\right]^{s} P_{E}(n)^{n-s} .
$$

The number of back-logged users, denoted by $X_{k}$ in the system can be modeled by a Markov chain. The equilibrium transition matrix is defined as $\mathbf{P}=\left[P_{i j}\right]$, where $P_{i j}$ is the one-step transition probability, given by

$$
P_{i j}=P\left\{X_{k+1}=j \mid X_{k}=i\right\} .
$$


Let the total number of transmitters be $N$. Using the same approach as in [4], we evaluate the transition probability as

$$
\begin{aligned}
P_{i j}=\sum_{n=0}^{N} & \sum_{s=\max (0, i-j)}^{\min (K, n, n-j+i)}\left(\begin{array}{c}
i \\
n-j+i-s
\end{array}\right) p_{R}^{n-j+i-s}\left(1-p_{R}\right)^{j-n+s} \\
& \times\left(\begin{array}{c}
N-i \\
j-i+s
\end{array}\right) p_{0}^{j-i+s}\left(1-p_{0}\right)^{N-j-s} P_{S}(s \mid n) .
\end{aligned}
$$

The stationary distribution of this Markov chain, i.e., $\pi_{i}=P\left\{X_{k}=i\right\}$, can be calculated by $\pi=\pi \mathbf{P}, \quad \sum_{i=0}^{N} \pi_{i}=$ 1. Throughput is defined as

$$
\begin{aligned}
\beta=E[S] & =E\left[E\left[S \mid N_{T}\right]\right] \\
& =\sum_{n=0}^{N}\left(\sum_{s=0}^{n} s P_{S}(s \mid n)\right) P_{N_{T}}(n)
\end{aligned}
$$

where the probability of transmitting $n$ packets in the system is given by

$$
\begin{aligned}
P_{N_{T}}(n) & =P\left\{N_{T}=n\right\}=\sum_{i=0}^{N} P\left\{N_{T}=n \mid X_{k}=i\right\} \pi_{i} \\
& =\sum_{i=0}^{N}\left[\sum_{m=\max (0, n-i)}^{\min (n, N-i)}\left(\begin{array}{c}
i \\
n-m
\end{array}\right) p_{R}^{n-m}\left(1-p_{R}\right)^{i-n+m}\right. \\
& \left.\times\left(\begin{array}{c}
N-i \\
m
\end{array}\right) p_{0}^{m}\left(1-p_{0}\right)^{N-i-m}\right] \pi_{i}
\end{aligned}
$$

The average delay that each packet experience can be evaluated by [4] $D=\frac{N}{\beta}-\frac{1}{p_{0}}$. When the number of users is large and the probability of retransmission and new packet transmission is small, the number of transmitted packets can be modeled by a Poisson distribution [2], i.e., $P_{N_{T}}(n)=\frac{y^{n}}{n !} e^{-y}$, where $y$ is the arrival rate. In this case the throughput is given by

$$
\beta=E[S]=\sum_{n=0}^{N}\left(\sum_{s=0}^{n} s P_{S}(s \mid n)\right) \frac{y^{n}}{n !} e^{-y} .
$$

In the following we use the above analytical framework to evaluate the delay and throughput in slotted ALOHA and CDMA slotted ALOHA networks using antenna arrays.

\section{Slotted ALOHA Networks}

In a non-spread spectrum system, using an $M$-element antenna array, at most $M$ simultaneous packets can be captured by the antenna array. The probability of packet error can be written as

$$
P_{E}(n)=\left\{\begin{array}{ll}
1 & n \leq M \\
0 & n>M
\end{array},\right.
$$

and the probability of successful transmission is given by

$$
P_{S}(s \mid n)= \begin{cases}P_{A}(s \mid n) & n \leq M, \quad s \leq M \\ 0 & \text { otherwise }\end{cases}
$$

That is, when there are more packets than the number of array elements all transmissions are unsuccessful. In order to circumvent this problem we send acknowledgments to a maximum of $M$ packets, and therefore the extra packets are blocked and only $M$ of them are transmitted. In this case the probability of successful transmission is given by

$$
P_{S}(s \mid n)=\left\{\begin{array}{ll}
P_{A}(s \mid n) & s \leq M, \quad s \leq n \\
0 & \text { otherwise }
\end{array} .\right.
$$

\section{CDMA slotted ALOHA Networks}

In the following we will evaluate the performance of a CDMA siotted ALOHA system using adaptive beamforming. The effect of call admission on delay and throughput is also evaluated. Assume each receiver uses an antenna array with $M$ elements and the processing gain is $\eta$. The SINR at the output of the beamformer is given by (1). The packet error probability in a system with BPSK/DS modulation:

$$
P_{b}(n)=Q\left(\sqrt{\frac{M \eta P}{\sigma^{2}+(n-1) P}}\right)
$$

where $Q(x)$ is the integral of the Gaussian pdf tail. If we use an e-error-correcting code, the probability of packet error, which is $P_{E}(n)$, is given by

$$
P_{E}(n)=1-\sum_{j=0}^{e}\left(\begin{array}{l}
L \\
j
\end{array}\right) P_{b}(n)^{j}\left(1-P_{b}(n)\right)^{L-j}
$$

We use a coding rate bound given by $[9] r=1-\frac{11 e}{L}$. Using (2) or (3) we evaluate the success probability, and using (4), we calculate the throughput. The effective throughput is defined as $\beta_{\text {eff }}=r \beta$. The SINR when $N$ packets are transmitted is given by

$$
\gamma=\frac{M \eta P}{(N-1) P}
$$

where we ignored the thermal noise at the receiver. Now assume using a call admission mechanism, we can prevent the acceptance of excess packets. For this purpose, the base station evaluates the SINR and, should it be less than a threshold, it will limit the number of transmitted packets to maintain the SINR above the threshold. The maximum number of users is then given by

$$
N_{\max }(m)=\left\{\begin{array}{ll}
m & m<\frac{M \eta}{\hat{n}}+1 \\
\frac{M}{\gamma}+1 & m \geq \frac{\hat{M}}{\gamma}+1
\end{array} .\right.
$$

Base station sends the transmit permission to $N_{\max }$ successfully acquired transmitters, and only packets transmitted by those transmitters will contribute to the cochannel interference. Therefore, the SINR is guaranteed to be at least $\gamma$. In order to evaluate the throughput, we evaluate $P_{b}(n)$ by $(8) . P_{E}(n)$ is given by:

$P_{E}(n)=1-\sum_{j=0}^{e}\left({ }_{j}^{L}\right) P_{b}\left(N_{\max }(n)\right)^{j}\left(1-P_{b}\left(N_{\max }(n)\right)\right)^{L-j}$.

The average throughput is expressed as

$$
\beta_{e f f}=r \sum_{n=1}^{N} \sum_{s=0}^{n} s P_{S}\left(s \mid N_{\max }(n)\right) P_{N_{T}}(n) .
$$




\section{MC-CDMA WITH ADAPTIVE ARRAYS}

The block diagram of a MultiCode CDMA (MC-CDMA) system is shown in Fig. 4. Transmitters convert the source data stream into parallel data signals and spread them with orthogonal Walsh codes. As a result, the interference between different bitstreams from the same user is almost zero. The spread streams are stacked to generate a composite synchronous CDMA signal. A PsuedoNoise (PN) sequence is superimposed on the spread signal that is used as a pilot for synchronization purposes. These sequences are assigned to each mobile during transmit permission stage. The pilot channel is modulated with a training sequence to perform beamforming.

The number of RA spreading codes $(K)$, and as a result the number of beamformers are less than the number of mobiles $(K<N)$. Therefore, we need to use a CDMA slotted ALOHA scheme for the request access channels. In this architecture each mobile has a queue. Mobile sends a Request Access (RA) only when a packet arrives at its empty queue. The RA contains the mobile signature and the number of packets in the queue. After the first transmission, if the queue is not empty, the RA is sent through piggybacking. RA packets are protected by error correcting codes. Coding increases the bandwidth of the signal and at the same time increases the probability of correct reception. Transmission is not successful, if more than one user chooses the same spreading code for the RA, or when multiuser interference causes an uncorrectable error. The mobile will then retransmit its packet, either with a constant probability $p_{r}$ or according to a harmonic backoff algorithm. In harmonic backoff algorithm, the mobile will retransmit the packet with probability $1 / n$, where $n$ is the number of unsuccessful attempts that mobile has made. Traffic channel capacity in the uplink is limited by multiuser interference. Using adaptive arrays, we can effectively reduce the cochannel interference (CCI) and increase capacity in the uplink. That is, adaptive arrays significantly increase the throughput in traffic and data channels. We use a training sequence as a beamforming preamble at the beginning of RA and data packets as illustrated in Fig. 5. We keep the beamforming weight vectors constant during the transmission of the RA or data.

At call admission each mobile is assigned to an orthogonal spreading code for the downlink. After successfully receiving the RA, the base station will send an transmit permission to the mobile using the assigned spreading code. The transmit permission consists of the number of packets allocated to that mobile for the current frame.

The mobile uses the same synchronous CDMA technique to spread and transmit its data packets to the base station. Assume mobile $m$ at most uses $N_{m}$ orthogonal codes to synchronously spread its packets. It should be noted that the interference between different codes of the same mobile is eliminated. However, the uplink packets of different mobiles are transmitted using asynchronous codes. As a result, the interference between different users limits the uplink capacity. After successfully receiving the packets, the base station sends a transmit acknowledgment to the mobile. At the acknowledgment, the base station marks the correctly received packets, and in the next frame mobiles retransmit the corrupted ones. These acknowledgments are transmitted using synchronous CDMA technique that eliminates inter-user in- terference.

At call setup, each mobile is associated with an average traffic rate $\bar{m}$, and a maximum number of packets per transmission $N_{m}$. The base station allocates uplink traffic channels to the mobiles according to a bandwidth on demand fair sharing algorithm [5].

\section{SIMULATION RESULTS}

Slotted ALOHA system performance is evaluated for a system setup as in [6]: $M=9, K=63, N=50$. The probability of successful transmission, with and without call admission, is calculated by (6) and (7) respectively. The stationary distribution for the total number of users is calculated by (5), and the throughput is given by (4). The throughput and delay as functions of the probability of new transmission are shown in Fig. 6(a) and (b) respectively. At low traffic, the throughput is linearly increased with traffic load. As the traffic increases, collision and interference levels increase and as a result throughput will be reduced significantly. Using an array with four elements, the maximum throughput of 1.5 is achieved with an average traffic rate of about 2 packets per slot. Using an array with 9 elements, the maximum achieved throughput is increased to 4.5 packets. Curves marked with Txprm show the case where call admission control is used. When we use a call admission mechanism the excess transmitters are prevented from transmission and the throughput does not drop at high traffic load. The maximum throughput in this case is almost equal to the number of array elements.

We simulate a CDMA slotted ALOHA system with beamforming in a network of 200 mobiles. The processing gain is $10 \mathrm{~dB}$. Throughput and delay as functions of $p_{0}$ are shown in Fig. 7(a) and (b), respectively. We use 10-errorcorrecting code, and the packet length is 1024 symbols. The simulation results show that by using an array with four elements we can increase the maximum throughput by a factor of 6 compared to a system with omnidirectional antennas. We have also evaluated the performance of the system when the number of active users is modeled by a Poisson process. The throughput for the processing gain of $17 \mathrm{~dB}$, packet length of 1024 , and with various coding rates, is shown in Fig. 8(a). The simulation results, where we have a call admission mechanism, is shown in Fíg. 8(b).

In order to evaluate the performance of a Multi-Beam MC-CDMA system, we simulate a system where each base station is equipped with four element antenna arrays. Each mobile divides the data stream into four equal rate sub-streams and uses four orthogonal codes to spread substreams. Each mobile is assigned to a unique $\mathrm{m}$-sequence as a pilot. The processing gain is $18 \mathrm{~dB}$ and the required SINR at the base station is $2.3 d B$. RA packets are coded with a coding rate of 0.9 . We use a slotted ALOHA protocol with harmonic backoff in the RA channels. We have distributed 300 mobiles in the system. The probability of new packet generation is varied, and the average delay is measured. First, we simulate an ideal system, where base stations have a full knowledge of the queues in the mobiles. Fig. 9(a) shows that the maximum capacity of network is increased by a factor equal to the number of elements in the array. Next we consider a non-ideal system with slotted ALOHA request access schemes with 
$\mathrm{K}=10$ request access spreading codes. Performance results is shown in Fig. 9(b). The simulation results show that the maximum capacity of network is increased by a factor equal to the number of elements in the array. In this case the array will also improve the throughput for the request access channels as well as traffic channels.

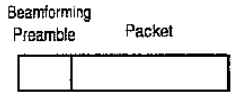

Figure 1: The structure of a packet.

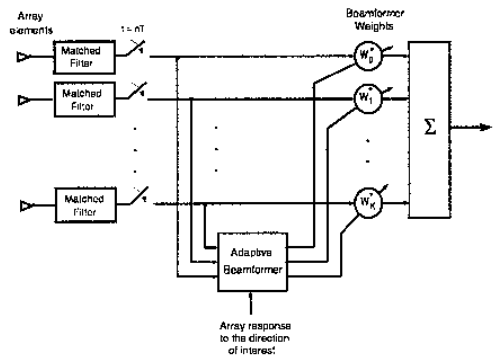

Figure 2: A diversity combining system.

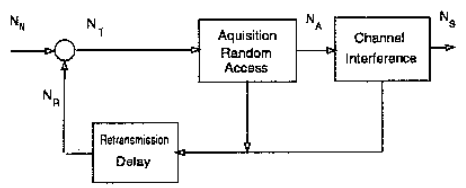

Figure 3: Block diagram of slotted ALOHA system.

\section{REFERENCES}

[1] N. Abramson, "The ALOHA system - another alternative for computer communications," In Fall Joint Comp. Conf., pp. 281-285. AFIPS Press, 1970.

[2] D. Raychaudhuri, "Performance analysis of random access packet-switched code division multiple access systems," IEEE Trans. Comm., vol. COM-29, No. 6, pp. 895-901, June 1981

[3] K. Joseph, and D. Raychaudhuri, "Throughput of unslotted direct sequence spread spectrum multiple access channels with block FEC coding," IEEE Trans. Comm., IEEE Trans. on Comm., vol. 41, No. 9, pp. 1373-1378, Sept. 1993.

[4] Wuyi Yue, "The effect of capture on performance of multichannel slotted ALOHA systems," IEEE Trans. on Comm., vol. 39, No. 6, pp. 818-822, June 1991.

[5] Z. Liu, and M. El Zerki, "Performance of DS-CDMA with slotted ALOHA random access for packet PCNs," ACM/Baltzer Wireless Network Journal", vol. 1, no. 1, pp. 1-16, February 1995.

[6] J. Ward, and R. T. Compton, Jr., "High throughput slotted ALOHA packet radio networks with adaptive arrays." IEEE Trans. on Comm., vol. 41, No. 3, pp. 460-470, March 1993.

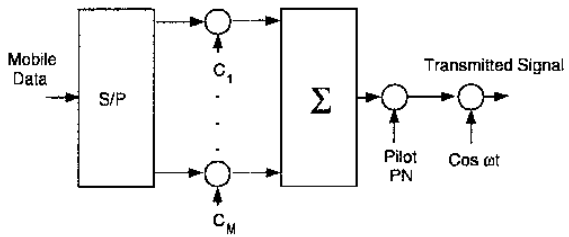

(a)

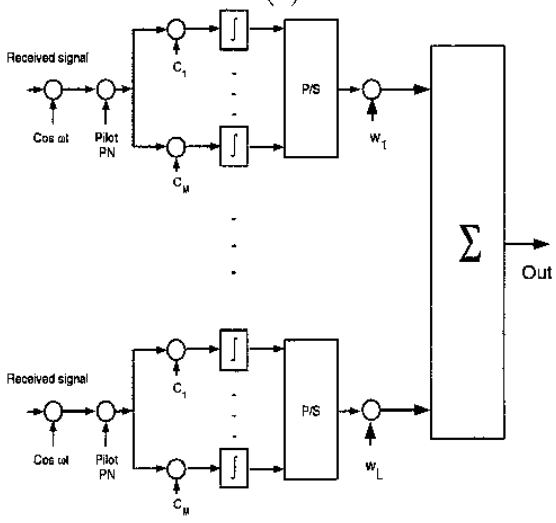

(b)

Figure 4: Multi-Beam MC-CDMA system; (a) Transmitter; (b) Receiver.

[7] B. Suard, A. Naguib, G. Xu, and A. Paulraj, "Performance analysis of CDMA mobile communication systerns using antenna array," in Proc. ICASSP' 93, vol. IV, Minneapolis, MN, pp. 153-156, April 1993.

[8] Chih Lin I, and R. D. Gitlin, "Multi-code CDMA wireless personal communications networks," In ICC'95 Conference Record, pp. 1060-1064, IEEE, June 1996.

[9] A. Plydoros, and J. Silverster, "Slotted random access spread-spectrum networks: an analytical framework," IEEE Jour. Slect. Areas Comm., vol. 5, No. 6, pp. 989-1002, July 1987.

[10] R. A. Monzingo and T. W. Miller, Introduction to Adaptive Arrays. New York, Wiley, 1980.

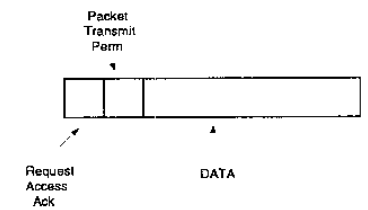

(a)

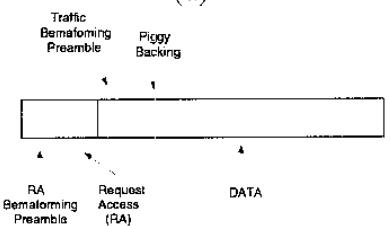

(b)

Figure 5: Multi-beam MC-CDMA frame structure; (a) Downlink; (b) Uplink. 


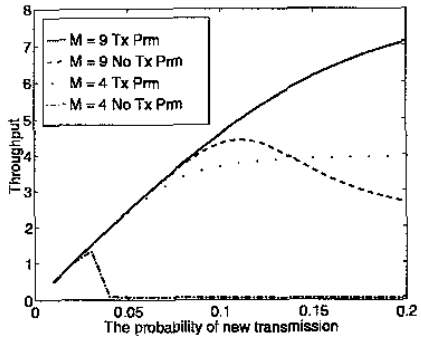

(a)

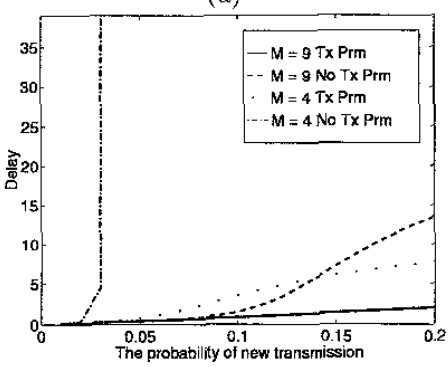

(b)

(a)

(b)

Figure 6: (a) Throughput; (b)Delay versus the probability of new transmission.

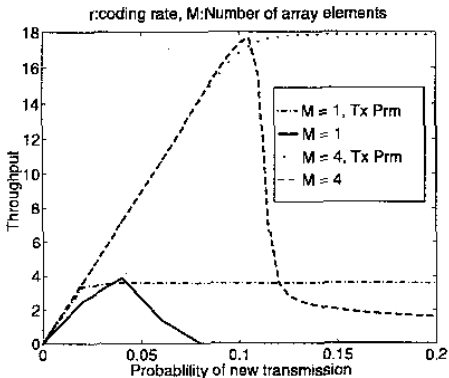

(a)

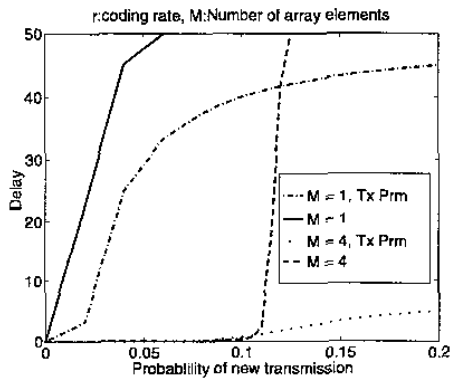

(b)

Figure 7: (a) Throughput; (b)Delay versus the probability of new transmission.

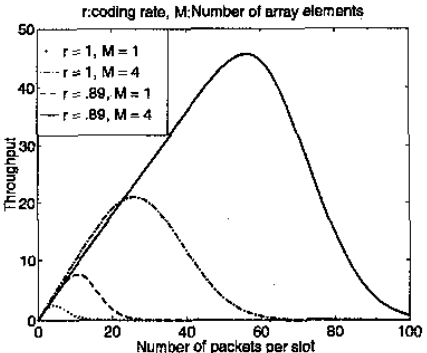

(a)

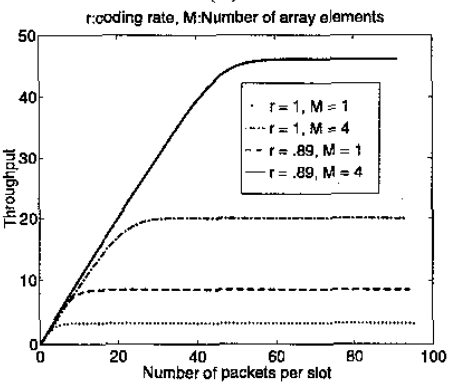

(b)

Figure 8: (a)Throughput versus load; (a) Without call admission; (b) With call admission.

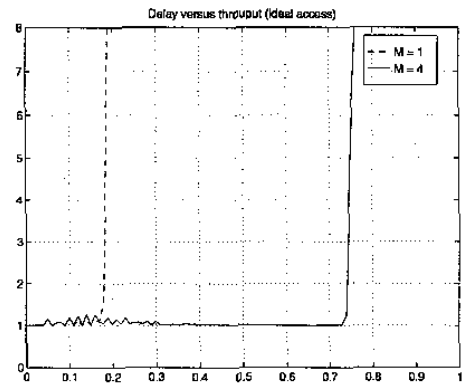

(a)

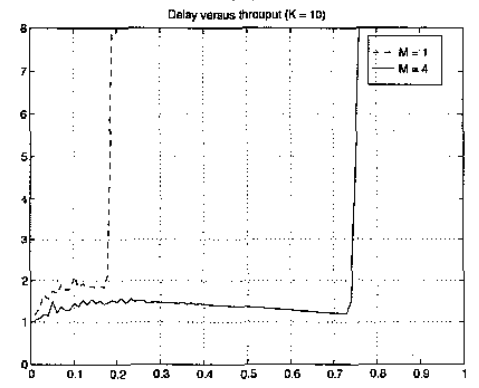

(b)

Figure 9: Delay; (a) Ideal case; (b) With $K=10$. 